Maria Helena Hasselmann 1 Michael E. Reichenheim 2

\section{Adaptação transcultural da versão em português da Conflict Tactics Scales Form $R$ (CTS-1), usada para aferir violência no casal: equivalências semântica e de mensuração}

\author{
Cross-cultural adaptation of the Portuguese \\ version of the Conflict Tactics Scales Form $R$ \\ (CTS-1) used to assess marital violence: \\ semantic and measurement equivalence
}

1 Departamento de Nutriçãoo Social, Instituto de Nutrição, Universidade do Estado do Rio de Janeiro. Rua São Francisco Xavier 524, 12 o andar, Rio de Janeiro, $R J$ 20559-900, Brasil. hasselm@ims.uerj.br 2 Instituto de Medicina Social, Universidade do Estado do Rio de Janeiro. Rua São Francisco Xavier 524, Pavilhão João Lyra Filho, 7 o andar, Blocos D e E, Rio de Janeiro, $R J$ 20559-900, Brasil. michael@ims.uerj.br

\begin{abstract}
This paper focuses on the cross-cultural adaptation of the Portuguese version of the Conflict Tactics Scales (CTS-1). Semantic equivalence was evaluated with regard to the referential meaning of words and the general connotative meaning of each item. Measurement equivalence between the Portuguese version and the original instrument was assessed by means of the version's psychometric properties, namely, intra-observer reliability, construct validity, internal consistency, and factor structure. For the different relationships, measurement agreement for physical aggression was moderate to substantial. Cronbach's a's were high for the physical and verbal aggression scales and low for the negotiation scale. As in the original instrument, factor analysis identified three dimensions, representing the negotiation, verbal aggression, and physical aggression scales, plus a severe physical aggression sub-scale. Although some problems still remain, the overall results suggest an adequate process of cross-cultural adaptation of the CTS-1, thus endorsing its use in the Brazilian setting.
\end{abstract}

Key words Domestic Violence; Validity; Questionnaires

Resumo Este artigo focaliza a adaptação transcultural da versão em português do instrumento Conflict Tactics Scales (CTS-1). A equivalência semântica foi avaliada no que diz respeito ao significado referencial dos termos/palavras e a acepção geral de cada item. A equivalência de mensuração entre a versão em português e o instrumento original foi apreciada por meio das propriedades psicométricas, tais como, sua confiabilidade teste-reteste, sua validade de constructo, sua consistência interna e sua estrutura de fatores. Para os diferentes relacionamentos, as concordâncias das mensurações sobre agressão física oscilaram entre moderada e substancial. Os $\alpha$ de Cronbach apresentaram-se elevados para as escalas de agressão física e verbal, e baixos para escala de argumentação. Da mesma forma que no instrumento original, a análise de fatores da versão da CTS-1 identificou três dimensões que representam as escalas de argumentação, de agressão verbal, de agressão física e mais uma subescala de agressão física grave. Ainda que algumas discrepâncias tenham sido notadas, como um todo os resultados sugerem uma adequação do processo de adaptação transcultural da versão da CTS-1 para a língua portuguesa, endossando seu uso na população brasileira.

Palavras-chave Violência Doméstica; Validade; Questionários 


\section{Introdução}

A violência familiar tem sido identificada como um problema social e de saúde pública em várias partes do mundo (Reiss Jr. \& Roth, 1993; Rossmann et al., 1999; Straus \& Gelles, 1995; Watts \& Zimmerman, 2002). No Brasil, o conhecimento sobre o tema, ainda que incipiente, vem crescendo nos últimos anos e estudos recentes sugerem que a violência familiar tem também grande expressividade em nossa sociedade (ABRAPIA, 1992; Deslandes, 1997, 1999; Deslandes et al., 2000; Ferreira et al., 1999; Ferreira \& Schramm, 2000; IBGE, 1999; Marques, 1986; Moura, 2002; MS, 1997; Reichenheim et al., 1999). Não obstante, o número de ações, quer preventivas ou de acompanhamento de suas vítimas, não tem sido dos mais abundantes. Isso pode ser explicado pela complexidade do fenômeno em si e pela dificuldade de seu reconhecimento. Na tentativa de contribuir para melhoria desta situação, torna-se necessário aprimorar as formas de aferição da violência familiar, tanto no sentido de sua suspeição, quanto no de sua identificação. Assim, é fundamental investir no desenvolvimento de instrumentos que possam ser utilizados nos serviços de saúde e em pesquisas.

A literatura nacional sobre concepção e desenvolvimento de instrumentos que visam a aferir violência familiar ainda é bastante escassa. Para o uso em estudos epidemiológicos, muito do que tem sido produzido apóia-se em instrumentos concebidos em outros idiomas, culturas e populações (Assis, 1992; Guedes, 1996; IBGE, 1999; Reichenheim \& Moraes, 2002). Ainda que tais instrumentos não tenham sido desenvolvidos com uma perspectiva internacional desde sua concepção, estes têm sido traduzidos para o português e utilizados sem uma adaptação formal à nossa cultura. A obtenção de êxito nessas situações é limitada, uma vez que as diferenças culturais e lingüísticas existentes entre populações e subgrupos populacionais não são levadas em consideração (Guillemin et al., 1993). Vários autores apontam que essa prática, além de gerar problemas de validade do instrumento, dificulta a comparação de resultados e perfis epidemiológicos entre diferentes localidades e populações (Alonso et al., 1990; Bravo et al., 1991; Herdman et al., 1998; Reichenheim \& Moraes, 2002). Nessa perspectiva, são imprescindíveis estudos sobre a adaptação transcultural de instrumentos.

O presente trabalho aborda o instrumento Conflict Tactics Scales Form R (CTS-1), desenvolvido no final da década de 70 (Straus, 1979). A CTS-1 objetiva mensurar as estratégias utili- zadas pelos membros da família para resolver possíveis desavenças e, indiretamente, captar uma situação de violência familiar. É composta por questões abarcando três táticas para lidar com conflitos: argumentação, que consiste no uso de discussão com uso de linguagem moderada e sensata; agressão verbal, valendo-se do uso de insultos e ameaças com intenção de, simbolicamente, machucar e agredir o outro; e agressão física, em que a força física explícita é usada. A CTS-1 original em inglês tem sido investigada desde sua concepção, e vários estudos apontam para uma baixa taxa de recusa; boa confiabilidade, principalmente para as escalas de violência física; e validade concorrente, de constructo e de conteúdo (Jouriles \& O'Leary, 1985; Straus, 1979; Straus \& Gelles, 1995).

O instrumento já vem sendo utilizado no nosso meio há alguns anos, quer na íntegra ou parcialmente (Assis, 1992; Guedes, 1996; IBGE, 1999). No entanto, algumas importantes etapas do processo de avaliação da adaptação transcultural não foram ainda contempladas. À luz do modelo proposto por Herdman et al. (1998), explicitado na seção seguinte, o presente estudo pretende focalizar dois aspectos centrais no estabelecimento da adequação do processo de adaptação transcultural da CTS-1, investigando especificamente as equivalências semântica e de mensuração entre o instrumento original e uma versão em português.

\section{Material \& métodos}

\section{Equivalência semântica}

Segundo Herdman et al. (1998), a equivalência semântica trata da transferência dos significados das palavras entre diferentes idiomas, e objetiva o alcance de efeitos similares em respondentes de culturas distintas. O significado, em si, pode estar articulado às idéias ou objetos do mundo aos quais uma ou várias palavras se referem (conotativo), ou ainda, se referir à acepção geral de cada item, captada por meio do instrumento original em comparação com a tradução (referencial). Visando a apreciar estas duas vertentes de significados, o presente trabalho se desenvolveu em quatro etapas: (1) tradução da escala de argumentação e uma revisão das escalas de agressão verbal e agressão física, as quais já vinham sendo utilizadas no Brasil; (2) teste da versão em um grupo de mães com filhos hospitalizados; (3) retradução ao idioma original e (4) apreciação da equivalência semântica propriamente dita. 
A primeira etapa consistiu na tradução da escala de argumentação por um especialista em língua inglesa e em uma apreciação realizada por dois pesquisadores da área de violência familiar quanto ao potencial entendimento pela população alvo dos itens já traduzidos. $\mathrm{Na}$ segunda etapa, a versão foi pré-testada em um grupo de mães com filhos hospitalizados. Procurou-se observar se havia correspondência literal entre a versão e o original, o nível de compreensão e a naturalidade de percepção das questões pela população alvo. Essa etapa foi realizada por entrevistadoras previamente treinadas e supervisionadas por um dos autores (M. H. H.). Subseqüentemente, a versão proposta foi retraduzida para o inglês por uma tradutora juramentada (terceira etapa).

Na última etapa, dois profissionais de nível superior, formados em Letras com especialização em inglês, realizaram uma apreciação formal da equivalência semântica. Adicionalmente, os autores do presente artigo apreciaram as avaliações feitas pelos especialistas, no intuito de clarificar certas incongruências encontradas e propor uma versão-síntese a ser subseqüentemente posta a teste (equivalência de mensuração).

Para avaliação de cada aspecto de equivalência realizada na quarta etapa foi usado um formulário específico. No formulário para apreciação do significado referencial optou-se por escalas visuais (Visual Analogue Scale-VAS), como opção de resposta (Streiner \& Norman, 1995). Para avaliação da acepção geral de cada pergunta utilizou-se uma escala diferencial com respostas discretas, a saber: inalterada, pouco alterada, muito alterada e completamente alterada.

\section{Equivalência de mensuração}

A equivalência de mensuração consiste em avaliar as medidas de confiabilidade e validade da versão do instrumento, comparando-as com as encontradas no instrumento original (Herdman et al., 1998). No caso da versão da CTS-1, foram apreciadas a confiabilidade intra-observador, a consistência interna de suas escalas, a estrutura de fatores e aspectos de validade de constructo.

Os dados analisados neste artigo são oriundos de um estudo caso-controle sobre a relação entre violência familiar e o aparecimento de desnutrição aguda grave (DAG) na infância (Hasselmann, 1996). Na análise da validade de constructo, apenas os controles foram utilizados, na medida em que a inclusão de casos (criança com DAG) poderia superestimar as asso- ciações investigadas. Essa decisão decorreu do conhecimento teórico e subseqüente evidência empírica de associações seletivamente maiores entre violência familiar e as variáveis eleitas para avaliar validade de constructo condições ambientais e de moradia; educação materna; e uso de drogas. Nas análises de confiabilidade, de consistência interna, e de fatores utilizaram-se casos e controles. Considerou-se que, por focalizarem o comportamento do instrumento em si, os resultados não seriam influenciados pela condição de outras variáveis, não sendo, portanto, necessário excluir nenhum subgrupo.

\section{- Confiabilidade intra-observador}

Foram entrevistados 54 sujeitos em duas ocasiões, em um intervalo variando de três dias a duas semanas. Foram avaliados os relacionamentos marido-esposa e esposa-marido. Quanto à classificação da violência no casal, considerou-se violentos todos os relacionamentos que apresentavam pelo menos uma resposta positiva aos itens relativos à escala de agressão física, tanto as menores $(k-m)$ quanto as graves $(n-s)$ (Straus \& Gelles, 1995). Esta escala, bem como as outras, pode ser observadas na Tabela 1 , apresentada na seção de resultados. A confiabilidade foi estimada pelo índice kappa (к) (Fleiss, 1981a), cuja interpretação seguiu a proposta de Shrout (1998). A concordância foi classificada como virtualmente ausente $(\kappa<0,1)$; fraca $(\kappa=0,10-0,40)$; discreta $(\kappa=0,41-0,60)$; moderada $(\kappa=0,61-0,80)$ e substancial $(\kappa=0,81-1,0)$.

\section{- Consistência interna}

A avaliação da consistência interna baseou-se no estimador $\alpha$ de Cronbach (Cronbach, 1951; Streiner \& Norman, 1995). O método de Feldt (1965) foi usado para o cálculo do limite inferior do intervalo de confiança. Analisaram-se os relacionamentos marido-esposa e esposa-marido, bem como o casal. As escalas da CTS-1 apreciadas foram as de argumentação (itens $a$ $c$ ), agressão verbal (itens $d$ - $f$ e $h$-j) e agressão física (itens $k$-s) (Straus \& Gelles, 1995). Nas três escalas foram contempladas três opções de respostas, a saber: não aconteceu, aconteceu algumas vezes nestes últimos doze meses e aconteceu várias vezes nestes últimos doze meses. Nesta análise foram utilizadas 517 observações.

\section{- Análise de fatores}

Nessa análise também se usou 517 casais. Os relacionamentos apreciados foram marido-es- 
Tabela 1

Equivalência semântica entre a versão em português das CTS-1 e o original em inglês.

\begin{tabular}{|c|c|c|c|c|c|c|c|}
\hline \multirow[t]{2}{*}{ Original } & \multirow[t]{2}{*}{ Traduzido } & \multirow[t]{2}{*}{ Retraduzido } & \multicolumn{2}{|c|}{ Aferidor 1} & \multicolumn{2}{|c|}{ Aferidor 2} & \multirow{2}{*}{$\begin{array}{c}\text { Síntese } \\
\text { Concordância } \\
(\%)\end{array}$} \\
\hline & & & $\begin{array}{l}\text { Concordância } \\
\text { (\%) }\end{array}$ & Avaliação & $\begin{array}{c}\text { Concordância } \\
\text { (\%) }\end{array}$ & Avaliação & \\
\hline $\begin{array}{l}\text { a) Discussed an } \\
\text { issue calmly }\end{array}$ & $\begin{array}{l}\text { a) Discutiu o problema } \\
\text { calmamente }\end{array}$ & $\begin{array}{l}\text { a) Calmly discussed } \\
\text { the problem }\end{array}$ & 80,0 & IN & 100,0 & IN & 90,0 \\
\hline $\begin{array}{l}\text { b) Got information to } \\
\text { back up your/ his/her } \\
\text { side of things }\end{array}$ & $\begin{array}{l}\text { b) Procurou conseguir } \\
\text { informações para } \\
\text { conhecer melhor } \\
\text { o modo de pensar } \\
\text { dele(a), ou o seu }\end{array}$ & $\begin{array}{l}\text { b) Tried to obtain } \\
\text { information about } \\
\text { his/her way of thinking, } \\
\text { or yours }\end{array}$ & 40,0 & MA & 95,0 & PA & 67,5 \\
\hline $\begin{array}{l}\text { c) Brought in, or tried } \\
\text { to bring in someone to } \\
\text { help settle things }\end{array}$ & $\begin{array}{l}\text { c) Trouxe, ou tentou } \\
\text { trazer alguém para } \\
\text { ajudar a acalmar } \\
\text { as coisas }\end{array}$ & $\begin{array}{l}\text { c) Brought, or tried to } \\
\text { get someone to help } \\
\text { calm things down }\end{array}$ & 100,0 & IN & 100,0 & IN & 100,0 \\
\hline $\begin{array}{l}\text { d) Insulted or swore } \\
\text { at him/her/ you }\end{array}$ & d) Xingou ou insultou & d) Swore or insulted & 100,0 & IN & 100,0 & IN & 100,0 \\
\hline $\begin{array}{l}\text { e) Sulked or refused } \\
\text { to talk about an issue }\end{array}$ & $\begin{array}{l}\text { e) Ficou emburrado. } \\
\text { Não falou mais } \\
\text { do assunto }\end{array}$ & $\begin{array}{l}\text { e) Sulked. Did not touch } \\
\text { the subject again }\end{array}$ & 100,0 & IN & 100,0 & IN & 100,0 \\
\hline $\begin{array}{l}\text { f) Stomped out of the } \\
\text { room, house, or yard }\end{array}$ & $\begin{array}{l}\text { f) Retirou-se do quarto, } \\
\text { da casa ou da área }\end{array}$ & $\begin{array}{l}\text { f) Left the room, the } \\
\text { house or the area }\end{array}$ & 90,0 & IN & 95,0 & PA & 92,5 \\
\hline g) Cried** & g) Chorou & g) Cried & 100,0 & IN & 100,0 & IN & 100,0 \\
\hline $\begin{array}{l}\text { h) Did or said } \\
\text { something to spite } \\
\text { him/her/you }\end{array}$ & $\begin{array}{l}\text { h) Fez ou disse coisas } \\
\text { só para irritar }\end{array}$ & $\begin{array}{l}\text { h) Did or said things } \\
\text { just to irritate }\end{array}$ & 100,0 & IN & 90,0 & PA & 95,0 \\
\hline $\begin{array}{l}\text { i) Threatened to hit } \\
\text { or throw something } \\
\text { at him/her/you }\end{array}$ & $\begin{array}{l}\text { i) Ameaçou bater ou } \\
\text { jogar coisas nele(a) } \\
\text { ou em você }\end{array}$ & $\begin{array}{l}\text { i) Threatened to hit or } \\
\text { throw things at him/her } \\
\text { or at you }\end{array}$ & 100,0 & IN & 100,0 & IN & 100,0 \\
\hline $\begin{array}{l}\text { j) Threw, smashed, hit, } \\
\text { or kicked something }\end{array}$ & $\begin{array}{l}\text { j) Destruiu, bateu, jogou } \\
\text { ou chutou objetos }\end{array}$ & $\begin{array}{l}\text { j) Destroyed, hit, threw } \\
\text { or kicked objects }\end{array}$ & 100,0 & IN & 100,0 & IN & 100,0 \\
\hline $\begin{array}{l}\text { k) Threw something } \\
\text { at him/her/you }\end{array}$ & $\begin{array}{l}\text { k) Jogou coisas sobre } \\
\text { ele/ela/(você) }\end{array}$ & $\begin{array}{l}\text { k) Threw things at } \\
\text { him/her/you }\end{array}$ & 100,0 & PA & 100,0 & IN & 100,0 \\
\hline $\begin{array}{l}\text { I) Pushed, grabbed, } \\
\text { or shoved him/her/you }\end{array}$ & $\begin{array}{l}\text { I) Empurrou ou agarrou } \\
\text { ele(a) ou você }\end{array}$ & $\begin{array}{l}\text { l) Pushed or grabbed } \\
\text { him/her/you }\end{array}$ & 100,0 & IN & 100,0 & IN & 100,0 \\
\hline $\begin{array}{l}\text { m) Slapped or spanked } \\
\mathrm{him} / \mathrm{her} / \text { you }\end{array}$ & $\begin{array}{l}\text { m) Deu tapa ou } \\
\text { bofetada nele(a) } \\
\text { ou em você }\end{array}$ & $\begin{array}{l}\text { m) Slapped or cuffed } \\
\text { him/her or you }\end{array}$ & 30,0 & IN & 80,0 & PA & 55,0 \\
\hline $\begin{array}{l}\text { n) Kicked, bit, or hit } \\
\text { him/her/you with a fist }\end{array}$ & $\begin{array}{l}\text { n) Chutou, mordeu ou } \\
\text { deu murro nele(a) ou } \\
\text { em você }\end{array}$ & $\begin{array}{l}\text { n) Kicked, bit or socked } \\
\text { him/her or you }\end{array}$ & 100,0 & IN & 100,0 & IN & 100,0 \\
\hline $\begin{array}{l}\text { o) Hit or tried to hit } \\
\text { him/her/you with } \\
\text { something }\end{array}$ & $\begin{array}{l}\text { o) Bateu ou tentou } \\
\text { bater nele(a) ou em } \\
\text { você com objetos }\end{array}$ & $\begin{array}{l}\text { o) Hit or tried to hit } \\
\text { him/her or you with } \\
\text { objects }\end{array}$ & 100,0 & IN & 100,0 & IN & 100,0 \\
\hline p) Beat him/her/you up & p) Espancou-o(a) ou você & p) Beat him/her or you & 100,0 & IN & 100,0 & IN & 100,0 \\
\hline $\begin{array}{l}\text { q) Burned or scalded } \\
\text { him/her; chocked him } \\
\text { or you/you }\end{array}$ & $\begin{array}{l}\text { q) Queimou-o(a); } \\
\text { estrangulou ou } \\
\text { sufocou-o ou você }\end{array}$ & $\begin{array}{l}\text { q) Burned him/her; } \\
\text { estrangled or suffocated } \\
\text { him or you }\end{array}$ & 100,0 & PA & 90,0 & PA & 95,0 \\
\hline $\begin{array}{l}\text { r) Threatened him/her/ } \\
\text { you with a knife or gun }\end{array}$ & $\begin{array}{l}\text { r) Ameaçou-o(a) ou } \\
\text { você com faca ou arma }\end{array}$ & $\begin{array}{l}\text { r) Threatened him/her or } \\
\text { you with a knife or gun }\end{array}$ & 100,0 & IN & 100,0 & IN & 100,0 \\
\hline $\begin{array}{l}\text { s) Used a knife or fired } \\
\text { a gun }\end{array}$ & $\begin{array}{l}\text { s) Usou faca ou arma } \\
\text { contra ele(a) ou você }\end{array}$ & $\begin{array}{l}\text { s) Used knife or gun against } \\
\text { him/her or you }\end{array}$ & st 100,0 & IN & 100,0 & IN & 100,0 \\
\hline Concordância total (\%) & & & 91,5 & & 97,3 & & 94,4 \\
\hline
\end{tabular}

$\mathrm{IN}=$ Inalterado; $\mathrm{PA}=$ Pouco alterado; $\mathrm{MA}=$ Muito alterado; $\mathrm{CA}=$ Completamente alterado. 
posa e esposa-marido. Buscando conhecer a performance da versão da CTS-1 quando utilizada para aferir o conflito no casal, também se implementou uma análise de fatores agregando as respostas das esposas sobre as suas reações em conflito e as dos respectivos companheiros. Para classificação da CTS-1 no casal utilizou-se o seguinte critério: não aconteceu em nenhum dos relacionamentos, aconteceu algumas vezes em pelo menos um relacionamento e aconteceu várias vezes em pelo menos um relacionamento.

$\mathrm{Na}$ análise de fatores utilizou-se o método de fatores principais iterados e rotação varimax (Rummel, 1988). Visando a comparação entre as características psicométricas da versão em português e as do instrumento original, decidiu-se especificar, a priori, quatro fatores para análise, a despeito da violação de alguns critérios sugeridos para escolha de fatores, tais como os critérios de Kaiser (autovalores $>1$ ), diagrama de scree ou o método de Lébart (Fleck \& Bourdel, 1998). Todos os itens foram analisados em três categorias, conforme explicitado na seção anterior.

\section{- Validade de constructo}

A validade de constructo foi examinada por meio das associações entre agressão física no casal e outras variáveis selecionadas pertinentes ao quadro teórico subjacente. A escolha dessas variáveis procurou refletir o conhecimento já consolidado na literatura sobre a validade de constructo da CTS-1 (Assis, 1992; Gelles, 1997; Straus, 1994; Straus \& Gelles, 1995). Como medida de associação entre as variáveis foi utilizada a razão de chances (odds ratio) (Fleiss, 1981b; Rosner, 1995). Para estas análises contou-se com 346 casais.

As variáveis estudadas foram: (a) anos de estudo formal da mãe (escala ordinal); (b) presença de geladeira e/ou fogão no lar, considerada um correlato de status sócio-econômico; (c) presença de argumentação entre o casal; (d) presença de agressão verbal marital e (e) uso de drogas ilícitas, incluindo suspeição de alcoolismo. O critério utilizado como indicador de uso de drogas foi baseado no consumo de drogas ilícitas pelo marido e/ou esposa nos últimos doze meses, mediante questionário NSDUQ Non-Student Drug Use Questionnaire - (Smart et al., 1981), combinando-se a resposta positiva do marido e/ou da esposa, a dois ou mais itens do questionário CAGE (Cut-down; Annoyed; Guilty \& Eye-opened) tradicionalmente usado para aferição de suspeição de alcoolismo (Mayfield et al., 1974).

\section{Resultados}

\section{Equivalência semântica}

Pode se notar na Tabela 1, que o instrumento original e sua retradução apresentam cerca de $68 \%$ dos itens com concordância completa; $21 \%$ entre $90 \%$ e $100 \%$ e apenas $10,5 \%$ com uma concordância menor do que $70 \%$. O percentual médio de concordância entre aferidores é de 94,4\%. A avaliação qualitativa do significado geral segue o mesmo padrão da apreciação quantitativa. De uma maneira geral, os significados se mostraram inalterados entre o original e a retradução. Os itens $b$ e $m$ foram os que apresentaram menor percentual de concordância.

\section{Equivalência de mensuração}

A Tabela 2 apresenta os resultados da confiabilidade das escalas de agressão física. Como um todo, observa-se que as estimações de ponto do coeficiente kappa estão acima de 0,70. Excetua-se o kappa referente ao relacionamento marido-esposa.

Conforme pode ser observado na Tabela 3, os $\alpha$ de Cronbach estão em torno de 0,70 para as escalas de agressão física e verbal, e entre 0,30 a 0,40 para escala de argumentação. O menor a encontrado concerne à escala de argumentação referente ao relacionamento esposamarido.

Na Tabela 3 também se encontram as estimações de consistência interna obtidas em um estudo usando o instrumento na língua inglesa (Straus \& Gelles, 1995). Observa-se que há semelhança entre este e a versão nas escalas de agressões verbal e física. Para a escala de argumentação, no entanto, os coeficientes encontrados no presente estudo são sistematicamente mais baixos do que os do original.

A Tabela 4 apresenta os resultados da análise de fatores por tipo de relacionamento e tam-

Tabela 2

Confiabilidade intra-observador da versão em português das CTS-1 para as escalas de agressão física.

\begin{tabular}{lccc}
\hline Relacionamentos & $\mathbf{n}$ & $\begin{array}{c}\text { Menor } \\
\text { Kappa (IC95\%) }\end{array}$ & $\begin{array}{c}\text { Grave } \\
\text { Kappa (IC95\%) }\end{array}$ \\
\hline Marido-esposa & 54 & $0,83(0,56-1,00)$ & $0,31(0,08-0,54)$ \\
Esposa-marido & 54 & $0,71(0,46-0,96)$ & $0,79(0,56-1,00)$ \\
\hline
\end{tabular}


Tabela 3

Consistência interna ( $\alpha$-Cronbach) da versão em português das CTS-1 e do instrumento original (Straus \& Gelles, 1995).

\begin{tabular}{|c|c|c|c|c|}
\hline Tipo de relaçãoa & CTS-1 & $\begin{array}{l}\text { Argumentação } \\
\text { (3 itens) }\end{array}$ & $\begin{array}{c}\text { Escalas } \\
\text { Agressão verbal } \\
\text { (6 itens) }\end{array}$ & $\begin{array}{c}\text { Agressão física } \\
\text { (9 itens) }\end{array}$ \\
\hline \multirow[t]{2}{*}{ Marido-esposa } & Versão em português & $0,38(0,30)^{b}$ & $0,73(0,70)$ & $0,82(0,80)$ \\
\hline & Original em inglêsc & 0,42 & 0,77 & 0,86 \\
\hline \multirow[t]{2}{*}{ Esposa-marido } & Versão em português & $0,34(0,26)$ & $0,74(0,71)$ & $0,76(0,73)$ \\
\hline & Original em inglês & 0,43 & 0,76 & 0,79 \\
\hline \multirow[t]{2}{*}{ Casal } & Versão em português & $0,36(0,28)$ & $0,82(0,79)$ & $0,83(0,81)$ \\
\hline & Original em inglês & 0,48 & 0,83 & 0,82 \\
\hline
\end{tabular}

a Para todas as relações as estimativas de consistência interna basearam-se em 517 observações.

b Estimativas retiradas de Straus \& Gelles (1995)

c Intervalo de confiança inferior a $95 \%$.

bém para o casal. As caselas hachuradas correspondem aos itens segundo as dimensões propostas no instrumento original em inglês, definindo as escalas de argumentação, agressão verbal, agressão física e uma subescala desta última. As caselas pontilhadas e os números em negrito se referem, respectivamente, aos itens que contribuem de maneira semelhante a dois fatores e aqueles que fazem parte do mesmo fator mas são originalmente colocados em outra(s) escala(s).

Quatro fatores são discriminados segundo o padrão na distribuição das cargas (loadings). Ainda que a Tabela 4 apresente muitos detalhes, os seguintes chamam a atenção. No relacionamento esposa-marido, o primeiro fator capta a escala de agressão física, incorporando também os itens $i$ ("ameaçou bater ou jogar coisas nele") e $j$ ("destruiu, bateu, jogou ou chutou objetos") da escala de agressão verbal do instrumento original. O segundo abarca os itens da escala de argumentação, agregando o item $f$ ("retirou-se do quarto, da casa ou da área"), que também contribui, em menor grau, para o quarto fator. O terceiro fator compreende uma das subescalas de violência, englobando os atos de agressão física potencialmente mais letais, como por exemplo, o item $r$ ("ameaçou/ usou faca ou arma contra a esposa”). O último fator se refere à maioria dos itens incluídos na escala de agressão verbal.

No relacionamento marido-esposa, o padrão é semelhante ao encontrado no recíproco, ainda que se perceba algumas inversões dos fatores especificados e certas diferenças quanto aos itens. Passam a pertencer ao primeiro fator os itens a ("discutiu o problema calmamente") e $l$ ("empurrou ou agarrou") que teoricamente deveriam se agregar, respectivamente, aos fatores referentes às escalas de argumentação e agressão física. Não obstante, o item $a$ tem sinal negativo, apontando a existência de uma correlação negativa entre a prática de diálogo e agressão verbal. Os itens $f$ ("retirou-se do quarto, da casa ou da área") e $m$ (“deu tapa ou bofetada") do fator 1 participam de maneira semelhante nos fatores 4 e 2, respectivamente.

Para a relação agregada (casal) há um padrão de distribuição de cargas e de retenção de fatores diferente do encontrado na análise por relacionamentos em separado. As discordâncias descritas não são mais detectadas. Os itens $i$ e $j$, por exemplo, agora se apresentam mais ligados à escala de agressão verbal. Por sua vez, as cargas do item $a$ se apresentam diliuídas entre os fatores 1, 2 e 3. O padrão de distribuição de cargas encontrado para o casal (agregado) é semelhante àquele descrito por Straus (1979).

É importante frisar que a análise de fatores da CTS original utiliza o formulário $\mathrm{N}$, a qual difere do R por excluir o item $q$. A troco de exploração, uma análise de fatores omitindo este item não mostrou grandes alterações na distribuição interna das cargas e estrutura de fatores. Por conseguinte, os demais resultados apresentados neste artigo atêm-se à CTS-1, formulário R.

Focalizando a validade de constructo, a Tabela 5 apresenta as associações entre algumas variáveis de interesse e agressão física no casal. Encontra-se uma associação positiva entre agressão física marital e uso de drogas e/ou álcool, dobrando naqueles casais que fazem uso dessas substâncias. A violência entre o casal 
Análise de fatores da versão em português da CTS-1.

\begin{tabular}{|c|c|c|c|c|c|c|c|c|c|c|c|c|}
\hline \multirow[t]{2}{*}{ Escalas/Item } & \multicolumn{4}{|c|}{ Relacionamento Esposa-Marido } & \multicolumn{4}{|c|}{ Relacionamento Marido-Esposa } & \multicolumn{4}{|c|}{ Relacionamento Casal } \\
\hline & f1 & f2 & f3 & $\mathrm{f4}$ & $f 1$ & $\mathrm{f} 2$ & f3 & $\mathrm{f} 4$ & $f 1$ & f2 & f3 & f4 \\
\hline \multicolumn{13}{|l|}{ Argumentação } \\
\hline a & $-0,166$ & 0,235 & $-0,081$ & 0,247 & $-0,359$ & 0,135 & 0,077 & 0,351 & $-0,045$ & 0,353 & 0,154 & $-0,167$ \\
\hline$b$ & 0,031 & 0,483 & $-0,093$ & $-0,051$ & $-0,139$ & 0,006 & $-0,047$ & 0,581 & 0,126 & 0,765 & $-0,009$ & 0,027 \\
\hline c & 0,189 & 0,459 & 0,075 & $-0,123$ & 0,131 & 0,010 & $-0,061$ & 0,226 & 0,344 & 0,190 & $-0,240$ & 0,150 \\
\hline \multicolumn{13}{|l|}{ Agressão verbal } \\
\hline d & 0,569 & 0,074 & 0,044 & $-0,491$ & 0,723 & $-0,254$ & $-0,099$ & $-0,004$ & 0,723 & 0,019 & $-0,149$ & 0,272 \\
\hline e & 0,245 & 0,195 & $-0,041$ & $-0,495$ & 0,457 & $-0,001$ & 0,042 & 0,315 & 0,616 & 0,255 & 0,088 & 0,020 \\
\hline$f$ & 0,146 & 0,434 & 0,052 & $-0,257$ & 0,379 & 0,087 & 0,037 & 0,360 & 0,505 & 0,250 & $-0,094$ & $-0,023$ \\
\hline $\mathrm{h}$ & 0,362 & 0,042 & 0,020 & $-0,470$ & 0,526 & $-0,041$ & 0,023 & 0,260 & 0,636 & 0,152 & $-0,071$ & 0,056 \\
\hline i & 0,750 & 0,123 & 0,108 & $-0,214$ & 0,649 & $-0,398$ & $-0,201$ & $-0,088$ & 0,672 & $-0,010$ & $-0,275$ & 0,404 \\
\hline j & 0,679 & $-0,143$ & $-0,012$ & $-0,256$ & 0,546 & $-0,256$ & $-0,180$ & 0,099 & 0,547 & 0,090 & $-0,221$ & 0,353 \\
\hline \multicolumn{13}{|c|}{ Agressão física menor } \\
\hline k & 0,763 & 0,045 & 0,126 & $-0,075$ & 0,327 & $-0,427$ & $-0,489$ & $-0,023$ & 0,463 & 0,091 & $-0,442$ & 0,418 \\
\hline 1 & 0,603 & 0,119 & 0,150 & $-0,119$ & 0,676 & $-0,318$ & $-0,214$ & $-0,089$ & 0,524 & $-0,012$ & $-0,326$ & 0,418 \\
\hline $\mathrm{m}$ & 0,717 & 0,149 & 0,085 & $-0,004$ & 0,547 & $-0,508$ & $-0,209$ & $-0,132$ & 0,472 & $-0,043$ & $-0,261$ & 0,564 \\
\hline \multicolumn{13}{|l|}{ Agressão física grave } \\
\hline $\mathrm{n}$ & 0,639 & $-0,044$ & 0,029 & 0,009 & 0,232 & $-0,817$ & 0,037 & $-0,027$ & 0,290 & 0,049 & $-0,111$ & 0,676 \\
\hline o & 0,693 & $-0,013$ & 0,125 & $-0,035$ & 0,277 & $-0,444$ & $-0,267$ & 0,027 & 0,355 & 0,103 & $-0,333$ & 0,452 \\
\hline $\mathrm{p}$ & 0,126 & 0,192 & $-0,034$ & 0,052 & 0,151 & $-0,815$ & $-0,029$ & 0,023 & 0,090 & $-0,023$ & 0,065 & 0,674 \\
\hline$q$ & 0,069 & 0,076 & 0,299 & 0,032 & 0,141 & $-0,474$ & $-0,092$ & $-0,064$ & 0,080 & $-0,069$ & $-0,126$ & 0,432 \\
\hline$r$ & 0,402 & $-0,153$ & 0,487 & $-0,091$ & 0,209 & $-0,213$ & $-0,481$ & $-0,026$ & 0,228 & $-0,016$ & $-0,654$ & 0,189 \\
\hline $\mathrm{s}$ & 0,155 & 0,039 & 0,712 & 0,014 & 0,153 & 0,089 & $-0,707$ & 0,024 & 0,102 & $-0,031$ & $-0,580$ & $-0,040$ \\
\hline $\begin{array}{l}\text { Variância explicada } \\
\text { pelo fator (\%) }\end{array}$ & 68 & 14 & 10 & 7 & 64 & 16 & 11 & 8 & 69 & 15 & 9 & 6 \\
\hline
\end{tabular}

em domicílios sem geladeira e/ou fogão é quatro vezes maior do que naqueles em que esses bens essenciais são encontrados, porquanto uma melhor condição sócio-econômica da família e mais elevados níveis de educação materna são fatores protetores.

Em relação às associações entre as próprias escalas da CTS-1, observa-se uma freqüência maior de agressão física nos casais que utilizam a agressão verbal como maneira de lidar com conflitos. A ocorrência de agressão física nos que não fazem uso de uma discussão moderada e sensata em momentos de desavenças é duas vezes e meia maior do que naqueles que praticam essa ação. Destaca-se que em todas as associações exploradas, os intervalos de confiança de $95 \%$ da estimativa de interesse excluíam o valor nulo.

\section{Discussão}

Segundo Herdman et al. (1998), antes que um instrumento possa ser traduzido para diferentes idiomas, é necessário avaliar se o constructo a ser mensurado é pertinente à nova cultura. A primeira etapa do processo de adaptação transcultural de instrumentos deve ser a apreciação das equivalências conceitual e de itens, no intuito de entender como diferentes populações expressam os conceitos e domínios a serem investigados e o modo com que as perguntas (itens) do instrumento os captam. Somente então é recomendado que se passe à avaliação das equivalências semântica, operacional, de mensuração e funcional.

Como visto, no momento em que se optou por utilizar a CTS-1, o instrumento já havia sido traduzido e utilizado por outros autores em investigações epidemiológicas no Brasil (Assis, 1992; Guedes, 1996; IBGE, 1999). Ainda que a 
Associações envolvendo selecionadas variáveis e as escalas das CTS-1.

\begin{tabular}{ll}
\hline Hipóteses & Relação testada \\
\hline $\begin{array}{ll}\text { Casais que usam drogas, incluindo álcool, utilizam mais violência física } \\
\text { RC (IC95\%) }\end{array}$ & $\begin{array}{l}\text { Uso de drogas, incluindo álcool } \\
\text { vs. violência física }\end{array}$ \\
$\begin{array}{l}\text { Casais que vivem em condições sócio-econômicas desfavoráveis } \\
\text { tendem apresentar mais violência física }\end{array}$ & $\begin{array}{l}\text { Ausência de geladeira e/ou fogão no lar } \\
\text { vs. violência física }\end{array}$ \\
$\begin{array}{l}\text { Casais com níveis de escolaridade maior tendem } \\
\text { a apresentar menos violência física }\end{array}$ & Educação materna vs. violência física \\
$\begin{array}{l}\text { Em casais onde ocorre agressão verbal também } \\
\text { existe mais violência física }\end{array}$ & Agressão verbal vs. violência física \\
$\begin{array}{l}\text { Em casais que não recorrem à tática de argumentação quando } \\
\text { há conflitos, tende a ocorrer mais violência física }\end{array}$ & Não discutiu o problema calmamente \\
\end{tabular}

metodologia proposta por Herdman et al. (1998) não tenha sido seguida à risca, alguns aspectos concernentes às equivalências conceitual e de itens acabaram sendo apreciadas neste trabalho. Empregando a edição já em uso corrente, avaliou-se a existência do conceito violência familiar em nossa sociedade mediante uma ampla revisão da literatura. Foram identificados vários estudos que corroboram a presença da violência familiar em nosso meio, inclusive quanto à semelhança das dimensões cobertas pela CTS-1 original (ABRAPIA, 1992; Deslandes, 1997; Ferreira et al., 1999; IBGE, 1999; Marques, 1986; MS, 1997; Soares, 1997). Mesmo assim, é importante considerar que, em função de não terem sido contempladas todas as etapas recomendadas por Herdman et al. (1998), maiores informações sobre as nuanças do vocabulário cotidiano não puderam ser apreendidas. A realização de grupos focais com a população alvo, por exemplo, poderia contribuir para um maior refinamento da versão do instrumento.

Como um todo, chama a atenção a adequada equivalência semântica, tanto do ponto de vista do significado referencial, quanto do geral (conotativo). No entanto, ocorreram algumas discrepâncias entre as avaliações dos aferidores, especificamente em relação aos itens $b$ e $m$. Neste, por exemplo, o termo spanked utilizado no instrumento original e retraduzido como cuffed, apesar de terem significados semelhantes (Vallandro, 1991), foram erroneamente avaliados como díspares na quarta etapa, possivelmente devido ao fato do último ser pouco usual. Não obstante, a tradução foi considerada adequada na apreciação subseqüente, apon- tando para a importância fundamental de uma avaliação crítica final do processo de traduçãoretradução.

Para as diversas relações estudadas, as concordâncias das mensurações sobre agressão física oscilaram entre moderada e substancial, exceto a referente ao relacionamento maridoesposa que, segundo os critérios de Shrout (1998), pode ser considerada fraca. Uma explicação para estes resultados, possivelmente recai sobre o receio dessas mulheres quanto às conseqüências negativas que a admissão e reafirmação de problemas de violência com seus companheiros pode acarretar.

Em relação à consistência interna, os coeficientes $\alpha$ são muito semelhantes aos achados por Straus \& Gelles (1995). A exceção está na escala de argumentação, na qual o $\alpha$ é ainda mais baixo do que no original. Straus \& Gelles (1995) concedem que a baixa consistência interna é devido ao pequeno número de itens (três) escolhidos para compor a referida escala, que, segundo os próprios autores, é claramente inadequado. Outros estudos focalizando sua ampliação merecem ser contemplados.

Mesmo que as demarcações para comparar os resultados de duas análises de fatores exploratórias não estejam bem estabelecidas, alguns autores sugerem critérios para guiar as avaliações de similaridade de estruturas fatoriais (Irvine \& Carroll, apud Behling \& Law, 2000). Entre estes, que o número de fatores extraídos do instrumento original e da versão seja idêntico e que a mesma proporção do total de variância seja observada para cada fator retido. A opção por reproduzir o estudo com a CTS-1 original, especificando a priori os quatro fatores a se- 
rem retidos na análise com a versão, impossibilitou que o primeiro critério fosse apreciado. No entanto, da mesma forma que no original, a análise de fatores da versão da CTS-1 também identificou três dimensões que representam as escalas de argumentação, de agressão verbal, de agressão física, além de mais uma subescala de agressão física grave.

O segundo critério, aparentemente, não foi inteiramente satisfeito a julgar pela diferente ordem de retenção dos fatores nas duas relações. Apesar de Straus (1979) não ter explicitado o quanto cada fator retido no instrumento original explicava da variância total, é possível inferir que uma relativa alteração das frações tenha ocorrido no presente estudo. Este achado, sem maiores qualificações, poderia estar indicando algum problema no processo de adaptação da CTS-1. Todavia, seria importante reconhecer a existência de uma certa labilidade dos dados acarretando em problemas intrínsecos à análise de fatores, em particular, no que tange à estrutura de covariâncias dos itens. A troco de ilustração, uma singela simulação alterando, em uma única observação do banco de dados na relação esposa-marido, as opções de respostas dos itens $q$, $r$ e $s$ de "nunca aconteceu" para "aconteceu várias vezes", leva a uma inversão da ordem de retenção dos fatores 2 e 3. Diante disso, talvez fosse mais prudente balizar a adequação do processo de adaptação apreciando este achado em conjunto com as outras evidências do estudo.

Levando em consideração o padrão de distribuição das cargas como critério de retenção de itens, visualiza-se uma estrutura de agregação semelhante àquela identificada com base na CTS-1 original (Straus, 1979). Contudo, algumas discrepâncias estão à vista. Para a relação esposa-marido, os itens $i$ e $j$ originalmente pertencentes à escala de agressão verbal, se agregam mais fortemente à de agressão física. Pode-se supor que algo esteja acontecendo em relação à interpretação da mulher sobre esses atos. Aparentemente, o fato de enfrentar o marido com ameaças de agressão física e, concretamente, destruir e jogar objetos, adquire em termos de intimidação, um significado semelhante ao de uma agressão física real. Investigações realizadas com diferentes populações e culturas, também verificaram uma maior contribuição desses itens para a escala de agressão física (Barling et al., 1987; Kumagai \& Straus, 1983; Moraes \& Reichenheim, 2002). Nota-se ainda que o item $l$ ("empurrou ou agarrou") se apresenta mais ligado à escala de agressão verbal quando a mulher passa a ser vítima, o que é algo um pouco inesperado, já que na CTS-1 original pertence à escala de agressão física. Estes achados talvez apontem para o caráter peculiar que alguns atos adquirem em nossa cultura, suspeita que certamente merece ser aprofundada.

Convém destacar que na presente investigação, por motivos logístico-operacionais, somente as esposas foram entrevistadas, respondendo por suas próprias reações em conflito e como proxis de seus companheiros. Isto difere de outros estudos. Em Barling et al. (1987), a CTS foi aplicada separadamente aos maridos e esposas. Mesmo que as análises se baseassem nas médias dos registros, ambos respondiam tanto sobre seus atos, quanto sobre os do outro. Archer (1999), em uma revisão meta-analítica sobre a confiabilidade da CTS, conclui que existe uma tendência do respondente em subregistrar comportamentos agressivos cometidos por si próprio. Straus (1979), comparando os resultados das análises de fatores de duas CTSs (formulários A e N), mostra que existem situações onde a interpretação da mulher sobre certos atos difere da do homem, fazendo com que os itens se agreguem em fatores diferentes na dependência da mulher ser a vítima ou perpetradora.

No presente estudo, o resultado da análise de fatores agregado por casal talvez sirva para corroborar esses pontos, pois os achados são comparáveis ao padrão de distribuição de cargas encontrado em Barling et al. (1987) e outros estudos realizados com a CTS em inglês para os relacionamentos em separado (Kumagai \& Straus, 1983; Straus, 1979). Classificar os itens das escalas agregando as respostas em não aconteceu em nenhum dos relacionamentos; aconteceu algumas vezes, pelo menos em um relacionamento; e aconteceu várias vezes, pelo menos em um relacionamento leva a um aumento de sensibilidade dos itens, o que parece indicar que a agregação esteja atuando como um elemento mitigador do problema de sub-registros e interpretação do respondente.

Os resultados obtidos na avaliação da validade de constructo também fornecem evidências da adequação da versão da CTS-1. Todas as associações apreciadas foram consistentes com o que prescreve o quadro teórico, bem como outras investigações empíricas. Estas mostram que a violência ocorre preferencialmente em casais de baixa escolaridade, vivendo em condições sócio-econômicas e ambientais precárias, dentre os quais o uso de álcool e drogas ilícitas são práticas comuns (Gelles, 1997; Straus et al., 1980; Straus \& Gelles, 1995). Serve de reforço à corroboração das hipóteses de que casais que tentam resolver desavenças valendo- 
se de ofensas verbais também são aqueles que praticam agressão física, ao contrário daqueles que optam pela argumentação. No entanto, não deve passar em branco que, devido ao fato dessas análises serem aninhadas a um estudo caso-controle com objetivos e quadro teórico bem definido, não foi possível investigar outros fatores relacionados à ocorrência de agressão física marital. A identificação de associações entre as diversas matizes da violência e outros corolários, tais como o nível de suporte social e desigualdade de estruturas de poder entre o casal, certamente teriam trazido mais robustez à apreciação da validade de constructo. De toda sorte, o que pôde ser estudado já permite uma avaliação satisfatória, sabido que as conjunções encontradas cobrem algumas das mais importantes descritas na literatura.

\section{Referências}

ABRAPIA (Associação Multiprofissional de Proteção à Infância e Adolescência), 1992. Maus Tratos Contra Crianças e Adolescentes. Proteção e Prevenção. Guia de Orientação para Profissionais de Saúde. Rio de Janeiro: ABRAPIA.

ALONSO, J.; ANTO, J. M. \& MORENO, C., 1990. Spanish version of Nottingham health profile: Translation and preliminary validity. American Journal of Public Health, 6:704-708.

ARCHER, J., 1999. Assessment of reliability of the Conflict Tactics Scales: A meta-analytic review. Journal of Interpersonal Violence, 14:1263-1289.

ASSIS, S. G., 1992. Violências, Crianças e Comportamentos: Um Estudo em Grupos Sociais Distintos. Relatório Final de Pesquisa. Rio de Janeiro: Centro Latino-Americano de Estudos sobre Violência e Saúde, Fundação Oswaldo Cruz.

BARLING, J.; O'LEARY, K. D.; JOURILES, E. M.; VIVIAN, D. \& MACEWEN, K. E., 1987. Factor similarity of the Conflict Tactics Scales across samples, spouses, and sites: Issues and implications. Journal of Family Violence, 2:37-54.

BEHLING, O. \& LAW, K. S., 2000. Translating Questionnaires and Other Research Instrument: Problems and Solutions. v. 7, n. 133. Thousand Oaks: Sage Publications.

BRAVO, M.; CANINI, G. J.; RUBIO-STIPEC, M. \& WOODBURY-FARINÃ, M., 1991. Cross-cultural adaptation of a psychiatric epidemiologic instrument: The Diagnostic Interview Schedule's adaptation in Puerto Rico. Culture, Medicine and Psychiatry, 15:1-18.
Os resultados satisfatórios do presente estudo, tanto no que diz respeito à equivalência semântica, quanto à de mensuração, sugerem a adequação do processo de adaptação transcultural da versão da CTS-1 para a língua portuguesa. No entanto, cumpre ressaltar que os dados analisados neste estudo são baseados em respostas de mães de baixo nível sócio-econômico que vivem no Município do Rio de Janeiro. Não deve ser descartada a possibilidade de que a estrutura fatorial e os resultados da confiabilidade venham a ser diferentes ao se aplicar a versão em outros grupos populacionais no Brasil. Esta expansão poderá gerar valiosas informações que contribuirão para ratificar a validade e confiabilidade do instrumento em geral, bem como consolidar sua adaptação transcultural, em particular.
CRONBACH, L. J., 1951. Coefficient alpha and the internal structure of test. Psychometrika, 16:297-334.

DESLANDES, S. F., 1997. Prevenir a Violência: Um Desafio para Profissionais de Saúde. Rio de Janeiro: Centro Latino-Americano de Estudos sobre Violência e Saúde Jorge Careli, Fundação Oswaldo Cruz.

DESLANDES, S. F., 1999. O atendimento às vítimas de violência na emergência: "Prevenção numa hora dessas?". Ciência \& Saúde Coletiva, 4:123-130.

DESLANDES, S. F.; GOMES, R. \& SILVA, C. M. F. P., 2000. Caracterização dos casos de violência doméstica contra a mulher atendidos em dois hospitais públicos do Rio de Janeiro. Cadernos de Saúde Pública, 16:129-137.

FELDT, L. J., 1965. The approximate sampling distribution of Kuder-Richardson reliability coefficient twenty. Psychometrika, 30:357-371.

FERREIRA, A. L. \& SCHRAMM, F. R., 2000. Implicações éticas da violência doméstica contra a criança para profissionais de saúde. Revista de Saúde Pública, 34:659-665.

FERREIRA, N. A.; GONÇALVES, H. S.; MARQUES, M. J. V. \& MORAES, S. R. S., 1999. A prevenção da violência contra criança na experiência do ambulatório de atendimento à família: Entraves e possibilidades de atuação. Ciência \& Saúde Coletiva, 4:123-130.

FLECK, M. P. A. \& BOURDEL, M. C., 1998. Método de simulação e escolha de fatores na análise dos componentes principais. Revista de Saúde Pública, 32:267-272. 
FLEISS, J. L., 1981a. Measuring nominal scales agreement among many raters. Psychology Bulletin, 76:378-382.

FLEISS, J. L., 1981b. Statistical Methods for Rates and Proportions. New York: John Wiley \& Sons.

GELLES, R. J., 1997. Intimate Violence in Families. London: Sage Publications.

GUEDES, L. G., 1996. Violência Doméstica: Prevalência e Fatores Associados em Famílias Atendidas num Serviço de Emergência Pediátrica. Dissertação de Mestrado, Rio de Janeiro: Universidade Federal do Rio de Janeiro.

GUILLEMIN, F.; BOMBARDIER, C. \& BEATON, D., 1993. Cross-cultural adaptation of health-related quality of life measures: Literature review and proposed guidelines. Clinical Epidemiology, 46: 1417-1432.

HASSELMANN, M. H., 1996. Violência Familiar e Desnutrição Severa na Infância: Modelo Teórico e Estudo de Confiabilidade dos Instrumentos. Dissertação de Mestrado, Rio de Janeiro: Instituto de Medicina Social, Universidade do Estado do Rio de Janeiro.

HERDMAN, M.; FOX-RUSHBY, J. \& BADIA, X., 1998. A model of equivalence in the cultural adaptation of HRQOL instruments: The universalist approach. Quality of Life Research, 7:323-335.

IBGE (Fundação Instituto Brasileiro de Geografia e Estatística), 1999. Resolução de Conflitos Domésticos e Violência Intrafamiliar nos Bairros da Tijuca e Maracanã. Rio de Janeiro: IBGE.

JOURILES, E. N. \& O'LEARY, K. D., 1985. Interspousal reliability of reports of marital violence. Journal of Consulting and Clinical Psychology, 53:419421.

KUMAGAI, F. \& STRAUS, M. A., 1983. Conflict resolution tactics in Japan, India, and USA. Journal of Comparative Family Studies, 3:376-392.

MARQUES, M. A. B., 1986. An Exploratory Study on Violence Against Children in a Favela in Rio de Janeiro. Ph.D. Thesis, New York: Columbia University.

MAYFIELD, D.; McLEOD, G. \& HALL, P., 1974. The CAGE questionnaires: Validation of new alcoholism screening instrument. American Journal of Psychiatry, 131:1121-1123.

MORAES, C. L. \& REICHENHEIM, M. E., 2002. Crosscultural measurement equivalence of the Revised Conflict Tactics Scales (CTS2) Portuguese version used to identify violence within couples. Cadernos de Saúde Pública, 18:783-796.

MOURA, A. T. M. S., 2002. Violência Familiar Contra Criança em um Serviço de Saúde: Gerando Informações para Ação. Dissertação de Mestrado, Rio de Janeiro: Universidade do Estado do Rio de Janeiro.

MS (Ministério da Saúde), 1997. Violência Contra Crianças e Adolescentes. Proposta Preliminar de Prevenção à Assistência à Violência Doméstica. Brasília: MS.
REICHENHEIM, M. E.; HASSELMANN, M. H. \& MORAES, C. L., 1999. Conseqüências da violência familiar na saúde da criança e do adolescente: Contribuições para a elaboração de propostas de ação. Ciência \& Saúde Coletiva, 4:109-121.

REICHENHEIM, M. E. \& MORAES, C. L., 2002. Buscando a qualidade das informações em pesquisas epidemiológicas. In: Caminhos do Pensamento: Epistemologia e Método (M. C. S. Minayo \& S. F Deslandes, org.), pp. 227-254, Rio de Janeiro: Editora Fiocruz.

REISS Jr., A. J. \& ROTH, J. A., 1993. Understanding and Preventing Violence. Washington, DC: National Academy Press.

ROSNER, B., 1995. Fundamentals of Biostatistics. Belmont: Duxbury Press.

ROSSMANN, B. B. R.; HUGHES, H. M. \& ROSENBERG, M. S., 1999. Children and Interparental Violence: The Impact of Exposure. Philadelphia: Brunner/Mazel.

RUMMEL, R. J., 1988. Applied Factor Analysis. $4^{\text {th }} \mathrm{Ed}$. Evanston: Northwest University Press.

SHROUT, P. E., 1998. Measurement reliability and agreement in psychiatry. Statistical Methods in Medical Research, 7:301-317.

SMART, R. G.; ARIF, A.; HUGHES, P.; MEDINA-MORA, M. E.; NAVARATNAM, V.; VARMA, V. K. \& WADUD, K. A., 1981. Drugs Use Among Non-Student Youth. Geneva: World Health Organization.

SOARES, B. M., 1997. Mulheres Invisíveis: Violência Familiar e Formações Subjetivas. Tese de Doutorado, Rio de Janeiro: Instituto Universitário de Pesquisas do Rio de Janeiro.

STRAUS, A. M. S.; GELLES, J. R. \& STEINMETZ, K. S. 1980. Behind Closed Doors. Violence in the American Family. New York: Anchor Press.

STRAUS, M. A., 1979. Measuring intrafamiliar conflict and violence: The Conflict Tactics (CT) Scales. Journal of Marriage and the Family, 41:75-88.

STRAUS, M. A., 1994. Beating the Devil Out of Them. Corporal Punishment in American Families. New York: Cambridge University Press.

STRAUS, M. A. \& GELLES, J. R., 1995. Physical Violence in American Families: Risk Factors and Adaptations to Violence in 8,145 Families. New Brunswick: Transaction Publishers.

STREINER, D. L. \& NORMAN, G. R., 1995. Health Measurement Scales. A Practical Guide to their Development and Use. Oxford: Oxford University Press.

VALLANDRO, L., 1991. Dicionário de Inglês: InglêsPortuguês/Português-Inglês. 16a Ed. São Paulo: Editora Globo.

WATTS, C. \& ZIMMERMAN, C., 2002. Violence against women: Global scope and magnitude. Lancet, 359:1232-1237.

Recebido em 1 de outubro de 2002

Aprovado em 28 de março de 2003 